\title{
THE TOEPLITZ THEOREM AND ITS APPLICATIONS TO APPROXIMATION THEORY AND LINEAR PDE'S
}

\author{
RONG-QING JIA
}

\begin{abstract}
We take an algebraic approach to the problem of approximation by dilated shifts of basis functions. Given a finite collection $\Phi$ of compactly supported functions in $L_{p}\left(\mathbb{R}^{s}\right) \quad(1 \leq p \leq \infty)$, we consider the shift-invariant space $S$ generated by $\Phi$ and the family $\left(S^{h}: h>0\right)$, where $S^{h}$ is the $h$ dilate of $S$. We prove that $\left(S^{h}: h>0\right)$ provides $L_{p}$-approximation order $r$ only if $S$ contains all the polynomials of total degree less than $r$. In particular, in the case where $\Phi$ consists of a single function $\varphi$ with its moment $\int \varphi \neq 0$, we characterize the approximation order of $\left(S^{h}: h>0\right)$ by showing that the above condition on polynomial containment is also sufficient.

The above results on approximation order are obtained through a careful analysis of the structure of shift-invariant spaces. It is demonstrated that a shiftinvariant space can be described by a certain system of linear partial difference equations with constant coefficients. Such a system then can be reduced to an infinite system of linear equations, whose solvability is characterized by an old theorem of Toeplitz. Thus, the Toeplitz theorem sheds light into approximation theory. It is also used to give a very simple proof for the well-known Ehrenpreis principle about the solvability of a system of linear partial differential equations with constant coefficients.
\end{abstract}

\section{INTRODUCTION}

In this paper we take an algebraic approach to the problem of approximation by dilated shifts of basis functions.

Given a compactly supported function $\varphi: \mathbb{R}^{s} \rightarrow \mathbb{C}$ and a complex-valued sequence $a$ on $\mathbb{Z}^{s}$, the semi-convolution $\varphi *^{\prime} a$ is the sum $\sum_{\alpha \in \mathbb{Z}^{s}} \varphi(\cdot-\alpha) a(\alpha)$. Let $J$ be a finite set, and let $\Phi=\left(\varphi_{j}\right)_{j \in J}$ be a finite collection of compactly supported functions from $\mathbb{R}^{s}$ to $\mathbb{C}$. We denote by $\mathscr{S}(\Phi)$ the linear space of all functions of the form $\sum_{j \in J} \varphi_{j} *^{\prime} a_{j}$, where $a_{j}(j \in J)$ are sequences on $\mathbb{Z}^{s}$. Then $S:=\mathscr{S}(\Phi)$ is shift-invariant in the sense that

$$
f \in S \Rightarrow f(\cdot-\gamma) \in S \quad \forall \gamma \in \mathbb{Z}^{s} .
$$

We call $S$ a finitely generated shift-invariant space.

Received by the editors February 9, 1994 and, in revised form, May 12, 1994; originally communicated to the Proceedings of the AMS by J. Marshall Ash.

1991 Mathematics Subject Classification. Primary 41A1 5, 41A25, 41 A63, 15A06, 35A99, 39A10.

Key words and phrases. Approximation order, shift-invariant spaces, infinite systems of linear equations, partial differential equations, partial difference equations.

This work was supported in part by the Canadian NSERC under Grant OGP 121336.

(C) 1995 American Mathematical Society 
Let us consider approximation in $L_{p}\left(\mathbb{R}^{s}\right)$ spaces $(1 \leq p \leq \infty)$. For a subset $G$ of $L_{p}\left(\mathbb{R}^{s}\right)$, the distance from $f$ to $G$, denoted by $\operatorname{dist}_{p}(f, G)$, is defined by

$$
\operatorname{dist}_{p}(f, G):=\inf _{g \in G}\|f-g\|_{p} .
$$

Let $\Phi$ be a finite collection of compactly supported functions in $L_{p}\left(\mathbb{R}^{s}\right)$. Let $S:=\mathscr{S}(\Phi) \cap L_{p}\left(\mathbb{R}^{S}\right)$ and $S^{h}:=\{g(\cdot / h): g \in S\} \quad(h>0)$. Given a nonnegative integer $r$, we say that $\mathscr{S}(\Phi)$ provides $L_{p}$-approximation order $r$ if, for any sufficiently smooth function $f \in L_{p}\left(\mathbb{R}^{s}\right)$,

$$
\operatorname{dist}_{p}\left(f, S^{h}\right)=O\left(h^{r}\right) .
$$

Denote by $\Pi_{r}$ the set of polynomials in $s$ variables of (total) degree $\leq r$. We agree that $\Pi_{-1}=\{0\}$. The Fourier transform of a function $\varphi \in L_{1}\left(\mathbb{R}^{s}\right)$ is defined by

$$
\hat{\varphi}(\xi):=\int_{\mathbb{R}^{s}} \varphi(x) e^{-i \xi \cdot x} d x, \quad \xi \in \mathbb{R}^{s},
$$

where $\xi \cdot x$ denotes the inner product of two vectors $\xi$ and $x$.

There has been an extensive study on approximation with shift-invariant spaces. Here we only mention three recent works. In [8] the author characterized the $L_{p}$-approximation order of $\mathscr{S}(\Phi)$ for the case when the space dimension $s=1$. When $\Phi$ consists of a single function $\varphi$, Ron in [15] gave a characterization of the $L_{\infty}$-approximation order of $\mathscr{S}(\Phi)$ as follows. If $\varphi$ is a compactly supported continuous function on $\mathbb{R}^{s}$ with $\hat{\varphi}(0) \neq 0$, then $\mathscr{S}(\varphi)$ provides $L_{\infty}$-approximation order $r$ if and only if $\mathscr{S}(\varphi) \supseteq \Pi_{r-1}$. We note that the method used in [15] cannot be applied to the case where $1 \leq p<\infty$. In [3], de Boor, DeVore, and Ron characterized the $L_{2}$-approximation order of $\mathscr{S}(\Phi)$. Their method works only for the $L_{2}$-norm. On the other hand, they allowed $\Phi$ to consist of arbitrary functions in $L_{2}\left(\mathbb{R}^{s}\right)$ (not necessarily of compact support).

One of our goals in this paper is to extend Ron's result to the case $1 \leq p \leq \infty$. One direction is relatively easy: If $\hat{\varphi}(0) \neq 0$ and $\mathscr{S}(\varphi) \supseteq \Pi_{r-1}$, then $\mathscr{S}(\varphi)$ provides $L_{p}$-approximation order $r$. Here we sketch the idea of the proof. Using the notion of commutator introduced in Chui, Jetter, and Ward [6], de Boor in [1] showed that $\hat{\varphi}(0) \neq 0$ and $\mathscr{S}(\varphi) \supseteq \Pi_{r-1}$ together imply that

$$
D^{\alpha} \hat{\varphi}(2 \pi \beta)=0 \quad \forall|\alpha|<r \text { and } \beta \in \mathbb{Z}^{s} \backslash\{0\} .
$$

If $\hat{\varphi}(0) \neq 0$ and (1.1) holds, then we say that $\varphi$ satisfies the Strang-Fix conditions of order $r$ (see [16] and [5]). Once we know that $\varphi$ satisfies the Strang-Fix conditions of order $r$, a quasi-interpolation technique can be used to produce an approximation scheme that provides the desired approximation order. See the paper [10] by Jia and Lei for an explicit construction of quasi-interpolants in the $L_{p}\left(\mathbb{R}^{s}\right)$ spaces $(1 \leq p \leq \infty)$. Also, see the recent survey paper [2] by de Boor on quasi-interpolants.

For the converse we are required to prove that $\mathscr{S}(\varphi) \supseteq \Pi_{r-1}$ if $\mathscr{S}(\varphi)$ provides $L_{p}$-approximation order $r$. In fact, we show that this is true for $\mathscr{S}(\Phi)$, where $\Phi$ consists of a finite number of compactly supported functions in $L_{p}\left(\mathbb{R}^{s}\right)$. A special case of this problem was discussed in [4] by de Boor and Höllig. To tackle this problem in the general setting, we need to investigate the structure of $\mathscr{S}(\Phi)$. It turns out that a shift-invariant space can be described by 
a certain system of linear partial difference equations with constant coefficients. Such a system then can be reduced to an infinite system of linear equations. Thus, we are led to the problem of solvaiblity of an infinite system of linear equations.

Let $A=\left(a_{i j}\right)_{i \in I, j \in J}$ be a matrix, where $I$ and $J$ are two (possibly infinite) sets and the entries $a_{i j}$ are in a field $K$. We assume that every row of $A$ is finitely supported, that is, for every $i \in I$, the set $J_{i}:=\left\{j: a_{i j} \neq 0\right\}$ is finite. Consider the system of linear equations:

$$
\sum_{j \in J} a_{i j} x_{j}=b_{i}, \quad i \in I,
$$

where the vector $\left(b_{i}\right)_{i \in I} \in K^{I}$ is given. We say that the system of linear equations (1.2) is consistent if it can be solved for $\left(x_{j}\right)_{j \in J} \in K^{J}$. The system of linear equations (1.2) is said to be compatible if, for every finitely supported vector $\lambda=\left(\lambda_{i}\right)_{i \in I} \in K^{I}$,

$$
\sum_{i \in I} \lambda_{i} a_{i j}=0 \quad \forall j \in J \Rightarrow \sum_{i \in I} \lambda_{i} b_{i}=0 .
$$

It is obvious that consistency implies compatibility. The converse implication is the content of the following theorem by Toeplitz. Its proof can be found in $[13$, p. 96].

Theorem 1 (Toeplitz). The system of equations (1.2) is consistent if and only if it is compatible.

In $\S 2$ we apply the Toeplitz theorem to the study of linear partial differential equations with constant coefficients and give a simple proof of the well-known Ehrenpreis fundamental principle on the solvability of such systems of linear pde's (see [7, Theorem 6.1]). In $\S 3$ we establish a similar result about linear partial difference equations with constant coefficients. Our approach is direct and elementary. Also, see [14] and [11] for some related results.

With the help of the result in $\S 3$, we are able to describe the structure of a finitely generated shift-invariant space. We say that a function $f$ is locally contained in $\mathscr{S}(\Phi)$ if, for any compact subset $E$ of $\mathbb{R}^{s}$, there exists an element $g_{E} \in \mathscr{S}(\Phi)$ such that $f$ and $g_{E}$ agree on $E$. In $\S 4$ we show that a function locally contained in $\mathscr{S}(\Phi)$ actually lies in it. In $\S 5$ this result is used to establish a lower bound for the approximation order. It is proved there that, if $\mathscr{S}(\boldsymbol{\Phi})$ provides $L_{p}$-approximation order $r$ for some $p \in[1, \infty]$, then $\mathscr{S}(\Phi)$ contains $\Pi_{r-1}$. Finally, if $\Phi$ consists of a single function $\varphi$ with $\hat{\varphi}(0) \neq 0$, then we prove that $\mathscr{S}(\varphi)$ provides $L_{p}$-approximation order $r$ for some $p \in[1, \infty]$ if and only if $\Pi_{r-1} \subseteq \mathscr{S}(\varphi)$.

\section{Partial Differential equations}

We use $K\left[X_{1}, \ldots, X_{s}\right]$ (resp. $\left.K \llbracket X_{1}, \ldots, X_{s} \rrbracket\right)$ to denote the ring of polynomials (resp. formal power series) in $s$ indeterminates $X_{1}, \ldots, X_{s}$ over $K$.

Let $\mathbb{N}$ be the set of nonnegative integers. Given $\alpha=\left(\alpha_{1}, \ldots, \alpha_{s}\right) \in \mathbb{N}^{s}$ and $\beta=\left(\beta_{1}, \ldots, \beta_{s}\right) \in \mathbb{N}^{s}, \alpha \leq \beta$ means $\alpha_{j} \leq \beta_{j}$ for all $j=1, \ldots, s$. Further, $\alpha !:=\alpha_{1} ! \cdots \alpha_{s} !$. The monomial $X_{1}^{\alpha_{1}} \cdots X_{s}^{\alpha_{s}}$ is denoted by $X^{\alpha}$. Moreover, the 
differential operator $D^{\alpha}$ on $K \llbracket X_{1}, \ldots, X_{s} \rrbracket$ is defined by the rule

$$
D^{\alpha}\left(\sum_{\beta \in \mathbb{N}^{s}} a_{\beta} X^{\beta}\right):=\sum_{\beta \geq \alpha} a_{\beta} \frac{\beta !}{(\beta-\alpha) !} X^{\beta-\alpha} .
$$

If $p=\sum_{\alpha \in \mathbb{N}^{s}} c_{\alpha} X^{\alpha}$ is a polynomial, then $p$ induces the differential operator $p(D)=\sum_{\alpha \in \mathbb{N}^{s}} c_{\alpha} D^{\alpha}$.

Let $f=\sum_{\beta \in \mathbb{N}^{s}} c_{\beta} X^{\beta}$ be an element in $K \llbracket X_{1}, \ldots, X_{s} \rrbracket$. We denote its constant term $c_{0}$ by $f(0)$. If $K$ is a field of characteristic 0 and if $D^{\alpha} f(0)=0$ for all $\alpha \in \mathbb{N}^{s}$, then $f=0$. If $K$ is of positive characteristic, then this conclusion does not hold in general.

Now let $\left(p_{i j}\right)_{i \in I, j \in J}$ be a matrix, where $I$ and $J$ are finite sets and each entry $p_{i j}$ is an element of $K\left[X_{1}, \ldots, X_{s}\right]$. Consider the system of partial differential equations:

$$
\sum_{j \in J} p_{i j}(D) u_{j}=f_{i}, \quad i \in I,
$$

where $f_{i}(i \in I)$ and $u_{j}(j \in J)$ are in $K \llbracket X_{1}, \ldots, X_{s} \rrbracket$.

The system of pde's (2.1) is said to be consistent if it can be solved for $\left(u_{j}\right)_{j \in J}$. It is said to be compatible if the following compatibility condition is satisfied: For $q_{i} \in K\left[X_{1}, \ldots, X_{s}\right](i \in I)$,

$$
\sum_{i \in I} q_{i} p_{i j}=0 \forall j \in J \Rightarrow \sum_{i \in I} q_{i}(D) f_{i}=0 .
$$

The following theorem was first proved by Ehrenpreis for the case when $K$ is the complex field $\mathbb{C}$. He raised the question whether his theorem could apply to an arbitrary field (see [7, p. 173]).

Theorem 2. Let $K$ be a field of characteristic 0 . Then the system of pde's (2.1) is consistent if and only if it is compatible.

Proof. Obviously, if $(2.1)$ is consistent, then it is compatible. Suppose now that (2.1) is compatible. We wish to show that it is consistent. The main idea of our proof is to reduce (2.1) to an infinite system of linear equations so that the Toeplitz theorem applies.

Since $K$ is a field of characteristic $0,\left(u_{j}\right)_{j \in J}$ satisfies the system of linear pde's in (2.1) if and only if

$$
D^{\alpha}\left(\sum_{j \in J} p_{i j}(D) u_{j}\right)(0)=D^{\alpha} f_{i}(0) \quad \forall \alpha \in \mathbb{N}^{s} \text { and } i \in I .
$$

Suppose

$$
\begin{aligned}
& f_{i}=\sum_{\alpha \in \mathbb{N}^{s}} \eta_{(i, \alpha)} X^{\alpha} / \alpha !, \quad i \in I, \\
& u_{j}=\sum_{\beta \in \mathbb{N}^{s}} \xi_{(j, \beta)} X^{\beta} / \beta !, \quad j \in J,
\end{aligned}
$$

and

$$
p_{i j}=\sum_{\gamma \in \mathbb{N}^{s}} c_{i j}(\gamma) X^{\gamma}, \quad(i, j) \in I \times J .
$$


Then $D^{\alpha} f_{i}(0)=\eta_{(i, \alpha)}$, and

$$
\begin{aligned}
& D^{\alpha}\left(p_{i j}(D) u_{j}\right)(0)=p_{i j}(D) D^{\alpha} u_{j}(0) \\
& \quad=p_{i j}(D)\left(\sum_{\beta \geq \alpha} \xi_{(j, \beta)} X^{\beta-\alpha} /(\beta-\alpha) !\right)(0)=\sum_{\beta \geq \alpha} c_{i j}(\beta-\alpha) \xi_{(j, \beta)} .
\end{aligned}
$$

Let

$$
a_{(i, \alpha),(j, \beta)}:= \begin{cases}c_{i j}(\beta-\alpha) & \text { if } \beta \geq \alpha, \\ 0 & \text { otherwise. }\end{cases}
$$

Thus the system of pde's $(2.1)$ is equivalent to the following system of linear equations:

$$
\sum_{(j, \beta) \in J \times \mathbb{N}^{s}} a_{(i, \alpha),(j, \beta)} \xi_{(j, \beta)}=\eta_{(i, \alpha)} \quad \forall(i, \alpha) \in I \times \mathbb{N}^{s} .
$$

It remains to show that the compatibility condition (2.2) implies the compatibility condition for the system of linear equations (2.4). For this purpose, let $\lambda \in K^{I \times \mathbb{N}^{s}}$ be such that $\lambda_{(i, \alpha)}=0$ except for finitely many $(i, \alpha) \in I \times \mathbb{N}^{s}$. Suppose

$$
\sum_{(i, \alpha) \in I \times \mathbb{N}^{s}} \lambda_{(i, \alpha)} a_{(i, \alpha),(j, \beta)}=0 \quad \forall(j, \beta) \in J \times \mathbb{N}^{s}
$$

Let

$$
q_{i}:=\sum_{\alpha \in \mathbb{N}^{s}} \lambda_{(i, \alpha)} X^{\alpha}, \quad i \in I
$$

Then

$$
\sum_{i \in I} q_{i} p_{i j}=\sum_{\beta \in \mathbb{N}^{s}} \sum_{\alpha \leq \beta} \lambda_{(i, \alpha)} c_{i j}(\beta-\alpha) X^{\beta} .
$$

From (2.3) and (2.5) we see that the sum $\sum_{\alpha \leq \beta} \lambda_{(i, \alpha)} c_{i j}(\beta-\alpha)=0$ for all $(j, \beta) \in J \times \mathbb{N}^{s}$; hence $\sum_{i \in I} q_{i} p_{i j}=0$ for all $j \in J$. Since (2.2) is satisfied, this implies

$$
\sum_{i \in I} q_{i}(D) f_{i}=0
$$

It follows that $\sum_{i \in I} q_{i}(D) f_{i}(0)=0$, that is,

$$
\sum_{(i, \alpha) \in I \times \mathbb{N}^{s}} \lambda_{(i, \alpha)} \eta_{(i, \alpha)}=0 .
$$

This shows that the system of linear equations (2.4) is compatible; hence it is consistent by the Toeplitz theorem. Therefore the system of pde's $(2.1)$ is consistent.

\section{PARTIAL Difference equations}

Let $K$ be a field. A mapping from $\mathbb{Z}^{s}$ to $K$ is called a $K$-sequence on $\mathbb{Z}^{s}$. Let $A$ be the linear space of all $K$-sequences on $\mathbb{Z}^{s}$. Given $\alpha \in \mathbb{Z}^{s}$, let $\tau^{\alpha}$ denote the difference operator on $S$ defined by $\tau^{\alpha} f:=f(\cdot+\alpha)$ for $f \in A$. A Laurent polynomial in $s$ indeterminates over $K$ has the form $\sum_{\alpha \in E} c_{\alpha} X^{\alpha}$, where $E$ is a finite subset of $\mathbb{Z}^{s}$. We use the notation $K\left[X_{1}, X_{1}^{-1}, \ldots, X_{s}, X_{s}^{-1}\right]$ 
to denote the ring of Laurent polynomials in $s$ indeterminates over $K$. If $p=\sum_{\alpha \in E} c_{\alpha} X^{\alpha}$ is a Laurent polynomial, then $p$ induces the difference operator $p(\tau)=\sum_{\alpha \in E} c_{\alpha} \tau^{\alpha}$.

Now let $\left(p_{i j}\right)_{i \in I, j \in J}$ be a matrix of Laurent polynomials, where $I$ and $J$ are finite sets. Consider the system of partial difference equations:

$$
\sum_{j \in J} p_{i j}(\tau) u_{j}=f_{i}, \quad i \in I,
$$

where $f_{i}(i \in I)$ and $u_{j}(j \in J)$ are in $A$.

The system of partial difference equations (3.1) is said to be consistent if it can be solved for $\left(u_{j}\right)_{j \in J}$. It is said to be compatible if the following compatibility condition is satisfied: For $q_{i} \in K\left[X_{1}, X_{1}^{-1}, \ldots, X_{s}, X_{s}^{-1}\right](i \in I)$,

$$
\sum_{i \in I} q_{i} p_{i j}=0 \forall j \in J \Rightarrow \sum_{i \in I} q_{i}(\tau) f_{i}=0 .
$$

Theorem 3. Let $K$ be an arbitrary field. Then the system of pde's (3.1) is consistent if and only if it is compatible.

Proof. Obviously, if (3.1) is consistent, then it is compatible. Suppose now that (3.1) is compatible. We wish to show that it is consistent. The proof is similar to that used in Theorem 2. We reduce (3.1) to an infinite system of linear equations and then apply the Toeplitz theorem to it.

Let

$$
\begin{aligned}
& \eta_{(i, \alpha)}=f_{i}(\alpha) \quad \forall(i, \alpha) \in I \times \mathbb{Z}^{s}, \\
& \xi_{(j, \beta)}=u_{j}(\beta) \quad \forall(j, \beta) \in J \times \mathbb{Z}^{s} .
\end{aligned}
$$

Suppose $p_{i j}=\sum_{\gamma \in \mathbb{Z}^{s}} c_{i j}(\gamma) X^{\gamma}$. Then

$$
p_{i j}(\tau) u_{j}(\alpha)=\sum_{\gamma \in \mathbb{Z}^{s}} c_{i j}(\gamma) u_{j}(\alpha+\gamma)=\sum_{\beta \in \mathbb{Z}^{s}} c_{i j}(\beta-\alpha) u_{j}(\beta) .
$$

This shows that (3.1) is consistent if and only if the following system of linear equations is consistent:

$$
\sum_{(j, \beta) \in J \times \mathbb{Z}^{s}} a_{(i, \alpha)(j, \beta)} \xi_{(j, \beta)}=\eta_{(i, \alpha)}, \quad(i, \alpha) \in I \times \mathbb{Z}^{s},
$$

where $a_{(i, \alpha),(j, \beta)}=c_{i j}(\beta-\alpha)$. The remainder of the proof is exactly the same as that of Theorem 2. If (3.1) is compatible, then (3.3) is also compatible; hence (3.3) is consistent by the Toeplitz theorem. We therefore conclude that (3.1) is consistent.

\section{SHIFT INVARIANT SPACES}

Let $S=\mathscr{S}(\Phi)$, where $\Phi=\left(\varphi_{j}\right)_{j \in J}$ is a finite collection of compactly supported functions from $\mathbb{R}^{s}$ to $\mathbb{C}$. Then the restriction of $S$ to $[0,1)^{s}$ is finite dimensional. Let $\Psi=\left(\psi_{i}\right)_{i \in I}$ be a basis for it. Suppose $a_{i} \quad(i \in I)$ are sequences on $\mathbb{Z}^{s}$. To $\left(a_{i}\right)_{i \in I}$ we associate a function $f$ on $\mathbb{R}^{s}$ as follows:

$$
f(x)=\sum_{i \in I} a_{i}(\beta) \psi_{i}(x-\beta) \text { for } x \in \beta+[0,1)^{s}, \beta \in \mathbb{Z}^{s} .
$$


We denote the function $f$ by $\left(a_{i}\right)_{i \in I}^{\Psi}$. Let $V$ be the linear space of all such functions. Then $S=\mathscr{S}(\Phi)$ is a linear subspace of $V$. In particular, each $\varphi_{j}$ has a representation $\left(a_{i j}\right)_{i \in I}^{\Psi}$, where each $a_{i j}$ is a finitely supported sequence on $\mathbb{Z}^{s}$.

A function $f \in V$ lies in $S=\mathscr{S}(\Phi)$ if and only if there are sequences $u_{j}$ $(j \in J)$ on $\mathbb{Z}^{s}$ such that

$$
f=\sum_{j \in J} \varphi_{j} *^{\prime} u_{j}
$$

Suppose $f=\left(f_{i}\right)_{i \in I}^{\Psi}$. If $x \in[0,1)^{s}+\beta$ for some $\beta \in \mathbb{Z}^{s}$, then $x-\alpha \in$ $[0,1)^{s}+\beta-\alpha$, and hence

$$
\varphi_{j}(x-\alpha)=\sum_{i \in I} a_{i j}(\beta-\alpha) \psi_{i}(x-\beta) .
$$

We see that (4.1) is equivalent to

$$
f(x)=\sum_{j \in J} \sum_{\alpha \in \mathbb{Z}^{s}} \varphi_{j}(x-\alpha) u_{j}(\alpha)=\sum_{j \in J} \sum_{\alpha \in \mathbb{Z}^{s}} \sum_{i \in I} a_{i j}(\beta-\alpha) \psi_{i}(x-\beta) u_{j}(\alpha) .
$$

But

$$
f(x)=\sum_{i \in I} f_{i}(\beta) \psi_{i}(x-\beta) .
$$

Since $\left(\psi_{i}\right)_{i \in I}$ forms a basis for $\left.S\right|_{[0,1)^{s}}$, it follows that

$$
f_{i}(\beta)=\sum_{j \in J} \sum_{\alpha \in \mathbb{Z}^{s}} a_{i j}(\beta-\alpha) u_{j}(\alpha)=\sum_{j \in J} \sum_{\alpha \in \mathbb{Z}^{s}} a_{i j}(\alpha) u_{j}(\beta-\alpha)
$$

for $i \in I$ and $\beta \in \mathbb{Z}^{s}$. This is equivalent to

$$
\sum_{j \in J} p_{i j}(\tau) u_{j}=f_{i}, \quad i \in I,
$$

where each $p_{i j}$ is the Laurent polynomial given by

$$
p_{i j}:=\sum_{\alpha \in \mathbb{Z}^{s}} a_{i j}(\alpha) X^{-\alpha} .
$$

Let $Q$ be the collection of those $\left(q_{i}\right)_{i \in I}$ for which

$$
q_{i} \in \mathbb{C}\left[X_{1}, X_{1}^{-1}, \ldots, X_{s}, X_{s}^{-1}\right] \quad(i \in I)
$$

and

$$
\sum_{i \in I} q_{i} p_{i j}=0 \quad \forall j \in J .
$$

Theorem 3 tells us that a function $f=\left(f_{i}\right)_{i \in I}^{\Psi}$ lies in $\mathscr{S}(\Phi)$ if and only if, for all $\left(q_{i}\right)_{i \in I} \in Q$,

$$
\sum_{i \in I} q_{i}(\tau) f_{i}=0
$$


Theorem 4. Let $\Phi=\left(\varphi_{j}\right)_{j \in J}$ be a finite collection of compactly supported functions from $\mathbb{R}^{s}$ to $\mathbb{C}$ and $S:=\mathscr{S}(\boldsymbol{\Phi})$. Then a function is contained in $S$ if and only if it is locally contained in $S$.

Proof. Let $f$ be a function locally contained in $S$. Assume that $f=\left(f_{i}\right)_{i \in I}^{\Psi}$. We wish to show that $f$ is contained in $S$. By the preceding remark, it suffices to show that, for every $\left(q_{i}\right)_{i \in I} \in Q, \sum_{i \in I} q_{i}(\tau) f_{i}=0$. Suppose $q_{i}=$ $\sum_{\gamma \in \mathbb{Z}^{s}} c_{i, \gamma} X^{\gamma}, i \in I$.

For $\alpha=\left(\alpha_{1}, \ldots, \alpha_{s}\right) \in \mathbb{Z}^{s}$ we use $|\alpha|_{\infty}$ to denote $\max _{1 \leq j \leq s}\left|\alpha_{j}\right|$. For a positive integer $N$, let $K_{N}:=\left\{\alpha \in \mathbb{Z}^{s}:|\alpha|_{\infty} \leq N\right\}$. Since each $q_{i}$ is a Laurent polynomial, there exists a positive integer $N$ such that $c_{i, \gamma}=0$ for all $(i, \gamma)$ with $i \in I$ and $\gamma \notin K_{N}$. Let $\alpha$ be a fixed element of $\mathbb{Z}^{s}$. Since $f$ is locally contained in $S$, there exists a $g \in S$ such that $g$ and $f$ agree on $\alpha+[-(N+1), N+1]^{s}$. It follows that $g$ and $f$ agree on $\beta+[0,1)^{s}$ for all $\beta \in \alpha+K_{N}$. Let $g=\left(g_{i}\right)_{i \in I}^{\Psi}$. Then $g_{i}(\beta)=f_{i}(\beta)$ for all $i \in I$ and $\beta \in \alpha+K_{N}$. Therefore we have

$$
\begin{aligned}
& \sum_{i \in I} q_{i}(\tau) f_{i}(\alpha)=\sum_{i \in I} \sum_{\gamma \in K_{N}} c_{i, \gamma} f_{i}(\alpha+\gamma) \\
& =\sum_{i \in I} \sum_{\gamma \in K_{N}} c_{i, \gamma} g_{i}(\alpha+\gamma)=\sum_{i \in I} q_{i}(\tau) g_{i}(\alpha)=0 .
\end{aligned}
$$

The last equality in the above is true since $g \in \mathscr{S}(\Phi)$. This shows that $\sum_{i \in I} q_{i}(\tau) f_{i}(\alpha)=0$ for all $\alpha \in \mathbb{Z}^{s}$; hence $\sum_{i \in I} q_{i}(\tau) f_{i}=0$, as desired.

\section{APPROXIMATION ORDER}

We are now in a position to consider approximation in $L_{p}\left(\mathbb{R}^{s}\right)$ spaces $(1 \leq$ $p \leq \infty)$. In this section, we use $I$ to denote the cube $[-1,1]^{s}$. If $E$ is a measurable subset of $\mathbb{R}^{s}$ and $1 \leq p<\infty$, then $\|f\|_{p}(E)$ denotes the quantity $\left(\int_{E}|f(x)|^{p} d x\right)^{1 / p}$. If $p=\infty$, then $\|f\|_{\infty}(E)$ denotes the essential supremum of $f$ over $E$.

Theorem 5. Let $\Phi=\left(\varphi_{j}\right)_{j \in J}$ be a finite collection of compactly supported functions in $L_{p}\left(\mathbb{R}^{s}\right)$. If $\mathscr{S}(\Phi)$ provides $L_{p}$-approximation order $r$ for some $p \in$ $[1, \infty]$, then $\mathscr{S}(\Phi)$ contains $\Pi_{r-1}$.

Proof. Our proof follows the lines of [12]. Write $S$ for $\mathscr{S}(\Phi)$. Suppose that $S$ provides $L_{p}$-approximation order $r$. We wish to prove that $S$ contains $\Pi_{r-1}$. By Theorem 4, it suffices to prove that $\Pi_{r-1}$ is locally contained in $S$. Suppose to the contrary that $\Pi_{r-1}$ is not locally contained in $S$. Then there is an integer $k$ with $0 \leq k \leq r-1$ such that $\Pi_{k-1}$ is locally contained in $S$ but $\Pi_{k}$ is not. By Theorem $4, \Pi_{k-1} \subseteq S$. Thus, there exists a nontrivial homogeneous polynomial $q$ of degree $k$ such that $q$ is not locally contained in $S$. For this $q$, there is a positive integer $m$ such that $\left.\left.q\right|_{m I} \notin S\right|_{m I}$. Let

$$
\delta:=\inf _{w \in S}\|q-w\|_{p}(m I) .
$$

Since $\left.S\right|_{m I}$ is finite dimensional, $\delta$ must be positive.

There exists a sufficiently smooth function $f \in L_{p}(\mathbb{R})$ such that $\left.f\right|_{I}=\left.q\right|_{I}$. Let $u \in S^{h}=\{g(\cdot / h): g \in S\} \quad(h>0)$. Then $u=w(\cdot / h)$ for some function 
$w \in S$. Hence

$$
\begin{aligned}
\| f- & u\left\|_{p}(I)=\right\| q-w(\cdot / h) \|_{p}(I) \\
& =h^{s / p}\|q(h \cdot)-w\|_{p}(I / h)=h^{s / p}\left\|h^{k} q-w\right\|_{p}(I / h),
\end{aligned}
$$

where we have used the fact that $q$ is homogeneous of degree $k$. Thus we obtain

$$
\|f-w(\cdot / h)\|_{p}(I)=h^{k+s / p}\left\|q-w / h^{k}\right\|_{p}(I / h) .
$$

If $p=\infty$ and $0<h<1 / m$, then (5.1) and (5.2) tells us that, for any $u=w(\cdot / h) \in S^{h}$,

$$
\|f-u\|_{\infty}(I)=h^{k}\left\|q-w / h^{k}\right\|_{\infty}(I / h) \geq h^{k} \delta .
$$

Thus, $S$ provides $L_{\infty}$-approximation order at most $k$ if $S$ does not contain $\Pi_{k}$.

Let us now consider the case $1 \leq p<\infty$. For $\beta \in \mathbb{Z}^{s}$ and $w \in S$ we have

$$
\|q-w\|_{p}(\beta+m I)=\|q(\cdot+\beta)-w(\cdot+\beta)\|_{p}(m I)=\|q-v\|_{p}(m I),
$$

where $v=q-q(\cdot+\beta)+w(\cdot+\beta)$. Since $S$ is shift-invariant, $w(\cdot+\beta) \in S$. Also, $q-q(\cdot+\beta) \in \Pi_{k-1} \subseteq S$. Hence $v \in S$, and by (5.1) we obtain

$$
\|q-w\|_{p}(\beta+m I)=\|q-v\|_{p}(m I) \geq \delta .
$$

Let $h=1 /[(2 n+1) m]$, where $n$ is a positive integer. Then

$$
\begin{aligned}
\| q- & w \|_{p}^{p}(I / h)=\int_{[-(2 n+1) m,(2 n+1) m]^{s}}|q-w|^{p} \\
& =\sum_{|\beta|_{\infty} \leq n} \int_{m I+2 m \beta}|q-w|^{p} \geq(2 n+1)^{s} \delta^{p}=(h m)^{-s} \delta^{p} .
\end{aligned}
$$

This together with (5.2) implies that, for any $u=w(\cdot / h) \in S^{h}$,

$$
\|f-u\|_{p}(I)=h^{k+s / p}\left\|q-w / h^{k}\right\|_{p}(I / h) \geq \delta m^{-s / p} h^{k} \text {. }
$$

The above estimate is true for $h=1 /[(2 n+1) m]$, where $n$ can be any positive integer. Since $k<r$, this contradicts the assumption that $S$ provides $L_{p}$ approximation order $r$.

Finally, we consider the case in which $\Phi$ consists of a single function $\varphi$.

Theorem 6. Let $\varphi$ be a compactly supported function in $L_{p}\left(\mathbb{R}^{s}\right) \quad(1 \leq p \leq \infty)$ such that $\hat{\varphi}(0) \neq 0$. Then the following statements are equivalent.

(a) $\mathscr{S}(\varphi)$ provides $l_{p}$-approximation order $r$.

(b) $\Pi_{r-1} \subset \mathscr{S}(\varphi)$.

(c) $\varphi$ satisfies the Strang-Fix conditions of order $r$.

The implication (a) $\Rightarrow$ (b) follows from Theorem 5 at once. With the assumption $\hat{\varphi}(0) \neq 0$, it is proved in [1] that (b) implies (c). The implication (c) $\Rightarrow$ (a) is well known. See [10] for an explicit construction of a quasiinterpolation scheme which provides the desired approximation order.

\section{ACKNOWLEDGMENT}

I am grateful to Professor Carl de Boor for his valuable comments on this paper. 


\section{REFERENCES}

1. C. de Boor, The polynomials in the linear span of integer translates of a compactly supported function, Constr. Approx. 3 (1987), 199-208.

2. _- Quasiinterpolants and approximation power of multivariate splines, Computation of Curves and Surfaces (W. Dahmen, M. Gasca, and C. A. Micchelli, eds.), Kluwer, Dordrecht, 1990, pp. 313-345.

3. C. de Boor, R. DeVore, and A. Ron, Approximation from shift-invariant subspaces of $L_{2}\left(\mathbb{R}^{d}\right)$, Trans. Amer. Math. Soc. 341 (1994), 787-806.

4. C. de Boor and K. Höllig, Bivariate box splines and smooth pp functions on a three direction mesh., J. Comput. Appl. Math. 9 (1983), 13-28.

5. C. de Boor and R. Q. Jia, Controlled approximation and a characterization of the local approximation order, Proc. Amer. Math. Soc. 95 (1983), 547-553.

6. C. K. Chui, K. Jetter, and J. D. Ward, Cardinal interpolation by multivariate splines, Math. Comp. 48 (1987), $711-724$.

7. L. Ehrenpreis, Fourier analysis in several complex variables, Wiley-Interscience, New York, 1970.

8. R. Q. Jia, A characterization of the approximation order of translation invariant spaces, Proc. Amer. Math. Soc. 111 (1991), 61-70.

9. __ A dual basis for the integer translates of an exponential box spline, Rocky Mountain J. Math. 23 (1993), 223-242.

10. R. Q. Jia and J. J. Lei, Approximation by multiinteger translates of functions having global support, J. Approx. Theory 72 (1993), 2-23.

11. R. Q. Jia, S. Riemenschneider, and Z. W. Shen, Solvability of systems of linear operator equations, Proc. Amer. Math. Soc. 120 (1994), 815-824.

12. J. J. Lei and R. Q. Jia, Approximation by piecewise exponentials, SIAM J. Math. Anal. 22 (1991), 1776-1788.

13. Yu. I. Lyubich, Functional Analysis. I, Encyclopaedia of Math. Sci., vol. 19, Springer-Verlag, Berlin, 1992.

14. U. Oberst, Multidimensional constant linear systems, Acta Appl. Math. 20 (1990), 1-175.

15. A. Ron, A characterization of the approximation order of multivariate spline spaces, Studia Math. 98 (1991), 73-90.

16. G. Strang and G. Fix, A Fourier analysis of the finite-element variational method, Constructive Aspects of Functional Analysis (G. Geymonat, ed.), C.I.M.E., II Ciclo 1971, Edizione Cremonese, Rome, 1973, pp. 793-840.

Department of Mathematics, University of Alberta, Edmonton, Alberta T6G 2G1, CANADA

E-mail address: jia@xihu.math.ualberta.ca 